\title{
ADMISSIBLE EXPONENTIAL REPRESENTATIONS AND TOPOLOGICAL INDICES FOR FUNCTIONS OF BOUNDED VARIATION
}

\author{
F. M. WRIGHT AND J. N. LING
}

\begin{abstract}
In this paper we first prove a theorem concerning the composition $\eta$ of an analytic complex-valued function $g$ in a region of the complex plane with a continuous complex-valued function $\phi$ of bounded variation on the closed interval $[a, b]$ of the real axis. We then relate this theorem to admissible exponential representations and topological indices introduced by Whyburn in his book Topological analysis. We also show how this theorem can be used to prove a result of interest in the study of the argument principle. Moreover, we look at the situation where $\phi$ is a complex-valued function of bounded variation but not necessarily continuous on a closed interval $[a, b]$ of the real axis, $p$ is a complex number not in the range of $\phi$, and $u$ is a complex-valued function on $[a, b]$ such that $e^{u(t)}=[\phi(t)-p]$ for $t$ in $[a, b]$. We present conditions for $u$ to be of bounded variation on $[a, b]$.
\end{abstract}

1. Introduction. In $\S 2$, we show that the composition $\eta$ of an analytic complex-valued function $g$ in a region $S$ of the complex plane with a continuous complex-valued function $\phi$ of bounded variation on a closed interval $[a, b]$ of the real axis is of bounded variation on $[a, b]$. We show further that $\int_{a}^{b} v(t) d \eta(t)=\int_{a}^{b} v(t) g^{\prime}(\phi(t)) d \phi(t)$ for any continuous complexvalued function $v$ on $[a, b]$. In $\S 3$, we use the result of $\$ 2$ to give a proof (patterned after that in $[1$, p. 56]) to show that if $\phi$ is a continuous complex-valued function of bounded variation on a closed interval $[a, b]$ of the real axis, $p$ is a complex number not in the range of $\phi$, and $u$ is a continuous complex-valued function on $[a, b]$ such that

$$
e^{u(t)}=\phi(t)-p, \quad t \in[a, b],
$$

then $u$ is of bounded variation on $[a, b]$. Whyburn [1] calls (1.1) an $a d-$ missible exponential representation of $[\phi(t)-p]$ on $[a, b]$, and he calls the

Received by the editors August 1, 1972 and, in revised form, January 8, 1973.

AMS (MOS) subject classifications (1970). Primary 33A90, 33A86, 26A45; Secondary 26A42, $26 \mathrm{~A} 54$.

Key words and phrases. Continuous function, bounded variation, admissible exponential representation, topological index, oriented Fréchet curve, argument principle, partition of a closed interval of the real axis, composite function, Stieltjes integral. 
complex number

$$
\mu(\phi, a, b, p)=u(b)-u(a)
$$

the topological index of the ordered pair $(\phi,[a, b])$ with respect to $p$. We also use the result of $\S 2$ to establish the integral formula

$$
\mu(\phi, a, b, p)=\int_{a}^{b}[\phi(t)-p]^{-1} d \phi(t)
$$

for the topological index (1.2). It then follows from a result in [2] or [3] that when $(\omega,[c, d])$ is any member of the oriented Fréchet curve for the complex plane containing the ordered pair $(\phi,[a, b])$, then $\mu(\omega, c, d, p)=$ $\mu(\phi, a, b, p)$. The preceding result generalizes $(1.5)$ of $[1, \mathrm{p} .59]$ for $\phi$ of bounded variation on $[a, b]$. In $\S 4$, we show how the result of $\S 2$ can be used to prove a result which relates to the argument principle.

In $\S 3$, we also look at the situation where $\phi$ is a complex-valued function of bounded variation but not necessarily continuous on a closed interval $[a, b]$ of the real axis, $p$ is a complex number not in the range of $\phi$, and $u$ is a complex-valued function on $[a, b]$ such that $(1.1)$ holds. We suppose that there is a positive real number $M$ such that $|\phi(t)-p| \geqq M$ for all $t$ in $[a, b]$. We suppose further that we have a positive real number $\varepsilon$ and a partition $\Delta$ of $[a, b]$ such that $\left|\operatorname{im} u\left(t^{\prime \prime}\right)-\operatorname{im} u\left(t^{\prime}\right)\right| \leqq \varepsilon$ whenever $t^{\prime}, t^{\prime \prime}$ are on one of the closed subintervals of $[a, b]$ determined by $\Delta$. We use a simple and direct proof to show that $u$ is of bounded variation on $[a, b]$. We observe that the condition here on the imaginary part of $u(t)$ holds when $u$ is continuous on $[a, b]$. Therefore, the result here, which is Theorem 3.3, generalizes the first result of $\$ 3$ mentioned above, which is Theorem 3.1. The proof we give of Theorem 3.3 provides an alternate approach to the one used in proving Theorem 3.1 via Theorem 2.1 and the Whyburn technique.

2. A theorem for functions of bounded variation. In this section, we establish the following theorem which we relate later to the study of admissible exponential representations, topological indices, and the argument principle.

THEOREM 2.1. Let $g$ be an analytic complex-valued function in an open subset $S$ of the complex plane. Let $\phi$ be a continuous complex-valued function of bounded variation on a closed interval $[a, b]$ of the real axis such that the range of $\phi$ is in $S$. Let $\eta(t)=g(\phi(t))$ for all $t$ in $[a, b]$. Then, $\eta$ is of bounded variation on $[a, b]$. Moreover, if $v$ is a continuous complex-valued function on $[a, b]$, then $\int_{a}^{b} v(t) d \eta(t)=\int_{a}^{b} v(t) g^{\prime}(\phi(t)) d \phi(t)$.

Proof. For each $z$ in $S$, let $\tilde{g}(z)=$ re $g(z)$ and $\hat{g}(z)=\operatorname{im} g(z)$. For each $t$ in $[a, b]$, let $\tilde{\phi}(t)=\operatorname{re} \phi(t)$ and $\hat{\phi}(t)=\operatorname{im} \phi(t)$, and let $\tilde{\eta}(t)=$ re $\eta_{(}(t)$ and 
$\hat{\eta}(t)=\operatorname{im} \eta(t)$. Let $T$ be the closure of a bounded and open set such that $T$ is a subset of $S$ and such that the range of $\phi$ is in the interior of $T$. Let $M$ be a positive real number such that $\left|\tilde{g}_{1}(z)\right| \leqq M,\left|\tilde{g}_{2}(z)\right| \leqq M$ for all $z$ in $T$. Let $\gamma$ be a positive real number such that for every $t$ in $[a, b]$, the $\gamma$-neighborhood of $\phi(t)$ is in $T$. Let $\delta$ be a positive real number such that $\mid \phi\left(t^{\prime \prime}\right)-$ $\phi\left(t^{\prime}\right) \mid<\gamma$ for every $t^{\prime}, t^{\prime \prime}$ in $[a, b]$ satisfying $\left|t^{\prime \prime}-t^{\prime}\right|<\delta$.

Let $t^{\prime}, t^{\prime \prime}$ be points of $[a, b]$ satisfying $\left|t^{\prime \prime}-t^{\prime}\right|<\delta$. Let $z^{\prime}=x^{\prime}+i y^{\prime}$ and $z^{\prime \prime}=x^{\prime \prime}+i y^{\prime \prime}$ be $\phi\left(t^{\prime}\right)$ and $\phi\left(t^{\prime \prime}\right)$, respectively, in rectangular form. Then,

$$
\begin{aligned}
\tilde{\eta}\left(t^{\prime \prime}\right)-\tilde{\eta}\left(t^{\prime}\right) & =\tilde{g}\left(z^{\prime \prime}\right)-\tilde{g}\left(z^{\prime}\right) \\
& =\tilde{g}_{1}\left((1-\theta) z^{\prime}+\theta z^{\prime \prime}\right)\left(x^{\prime \prime}-x^{\prime}\right)+\tilde{g}_{2}\left((1-\theta) z^{\prime}+\theta z^{\prime \prime}\right)\left(y^{\prime \prime}-y^{\prime}\right)
\end{aligned}
$$

for some real number $\theta$ satisfying $0<\theta<1$. It follows that

$$
\left|\tilde{\eta}\left(t^{\prime \prime}\right)-\tilde{\eta}\left(t^{\prime}\right)\right| \leqq M\left\{\left|\tilde{\varphi}\left(t^{\prime \prime}\right)-\tilde{\varphi}\left(t^{\prime}\right)\right|+\left|\hat{\phi}\left(t^{\prime \prime}\right)-\hat{\phi}\left(t^{\prime}\right)\right|\right\} .
$$

From this it follows that $\tilde{\eta}$ is of bounded variation on $[a, b]$. Similarly, $\hat{\eta}$ is of bounded variation on $[a, b]$. Thus, $\eta$ is of bounded variation on $[a, b]$.

Let $\Delta=\left\{a=t_{0}<t_{1}<t_{2}<\cdots<t_{n}=b\right\}$ be any partition of $[a, b]$ with norm less than $\delta$. For each integer $j=0,1,2, \cdots, n$, let $z_{j}=x_{j}+i y_{j}$ be the complex number $\phi\left(t_{j}\right)$ in rectangular form. For each integer $j=1,2$, $\cdots, n$, let $\theta_{j}$ be a real number satisfying $0<\theta_{j}<1$ such that

$$
\begin{aligned}
& \tilde{\eta}\left(t_{j}\right)-\tilde{\eta}\left(t_{j-1}\right) \\
& \quad=\tilde{g}_{1}\left(\left(1-\theta_{j}\right) z_{j-1}+\theta_{j} z_{j}\right)\left(x_{j}-x_{j-1}\right)+\tilde{g}_{2}\left(\left(1-\theta_{j}\right) z_{j-1}+\theta_{j} z_{j}\right)\left(y_{j}-y_{j-1}\right) .
\end{aligned}
$$

We may use the boundedness of $v(t)$ on $[a, b]$, the uniform continuity of $\tilde{g}_{1}$ and $\tilde{g}_{2}$ on $T$, and the continuity and rectifiability of $\phi$ on $[a, b]$ to show that

It then follows that

$$
\begin{aligned}
\lim _{\|\Delta\| \rightarrow 0} \sum_{j=1}^{n} v\left(t_{j}\right)\left[\tilde{g}_{1}((1\right. & \left.\left.-\theta_{j}\right) z_{j-1}+\theta_{j} z_{j}\right)\left(x_{j}-x_{j-1}\right) \\
& \left.+\tilde{g}_{2}\left(\left(1-\theta_{j}\right) z_{j-1}+\theta_{j} z_{j}\right)\left(y_{j}-y_{j-1}\right)\right] \\
= & \int_{a}^{b} v(t) \tilde{g}_{1}(\phi(t)) d \tilde{\phi}(t)+\int_{a}^{b} v(t) \tilde{g}_{2}(\phi(t)) d \hat{\phi}(t) .
\end{aligned}
$$

$$
\int_{a}^{b} v(t) d \tilde{\eta}(t)=\int_{a}^{b} v(t) \tilde{g}_{1}(\phi(t)) d \tilde{\phi}(t)-\int_{a}^{b} v(t) \hat{g}_{1}(\phi(t)) d \hat{\phi}(t) .
$$

Similarly,

$$
\int_{a}^{b} v(t) d \hat{\eta}(t)=\int_{a}^{b} \nu(t) \hat{g}_{1}(\phi(t)) d \tilde{\phi}(t)+\int_{a}^{b} \nu(t) \tilde{g}_{1}(\phi(t)) d \hat{\phi}(t) .
$$


Therefore,

$$
\int_{a}^{b} v(t) d \eta(t)=\int_{a}^{b} v(t) g^{\prime}(\phi(t)) d \phi(t) .
$$

This completes the proof of Theorem 2.1.

3. Topological indices. First we show how Theorem 2.1 can be used to prove the following result concerning the admissible exponential representation (1.1) when the function $\phi$ is continuous and of bounded variation on $[a, b]$. The proof we give is patterned after that given by Whyburn [1, p. 56].

THEOREM 3.1. Let $\phi$ be a cont inuous complex-valued function of bounded variation on a closed interval $[a, b]$ of the real axis. Let $p$ be a complex number not in the range of $\phi$. Let $u$ be a continuous complex-valued function on $[a, b]$ such that (1.1) holds. Then, $u$ is of bounded variation on $[a, b]$.

Proof. Consider first the case where there is a ray $L$ in the complex plane emanating from $p$ such that $L$ contains no points in the range of $\phi$. Let $S$ be the region consisting of all complex numbers $z$ not on $L$. There is an analytic complex-valued function $g$ in $S$ such that $e^{g(z)}=(z-p)$ for all $z$ in $S$. Let $\eta(t)=g(\phi(t))$ for all $t$ in $[a, b]$. It is apparent that $\eta$ is continuous on $[a, b]$, and we have from Theorem 2.1 that $\eta$ is also of bounded variation on $[a, b]$. It is clear that $e^{\eta(t)}=\phi(t)-p$ for all $t$ in $[a, b]$.

Next we drop the restriction about the ray $L$. Proceeding as in the proof of (1.2) on p. 56 of [1], we have that there is a continuous complex-valued function $\eta$ of bounded variation on $[a, b]$ such that $e^{\eta(t)}=\phi(t)-p$ for all $t$ in $[a, b]$.

There is a constant $k$ such that $u(t)=\eta(t)+k$ for all $t$ in $[a, b]$. Thus, $u$ is also of bounded variation on $[a, b]$.

This completes the proof of Theorem 3.1.

Next we show how Theorem 2.1 can be used to prove the following result which provides an integral formula for the topological index (1.2) when the function $\phi$ is continuous and of bounded variation on $[a, b]$.

THEOREM 3.2. Let $\phi$ be a continuous complex-valued function of bounded variation on a closed interval $[a, b]$ of the real axis. Let $p$ be a complex number not in the range of $\phi$. Then, $\mu(\phi, a, b, p)=\int_{a}^{b}[\phi(t)-p]^{-1} d \phi(t)$.

Proof. Let $u$ be a continuous complex-valued function of bounded variation on $[a, b]$ such that $(1.1)$ holds. Then,

$$
\int_{a}^{b}[\phi(t)-p]^{-1} d \phi(t)=\int_{a}^{b} e^{-u(t)} d\left[e^{u(t)}+p\right]=\int_{a}^{b} e^{-u(t)} e^{u(t)} d u(t)
$$

in view of Theorem 2.1. The desired result then follows.

This completes the proof of Theorem 3.2. 
The next result concerns the formula (1.1) when $\phi$ is a complex-valued function of bounded variation on the closed interval $[a, b]$ of the real axis such that $\phi$ is not necessarily continuous on $[a, b]$. This result provides conditions for $u$ to be of bounded variation on $[a, b]$ and extends Theorem 3.1. We give a simple, direct proof of Theorem 3.3 different from the proof above for Theorem 3.1.

THEOREM 3.3. Let $\phi$ be a complex-valued function of bounded variation on a closed interval $[a, b]$ of the real axis. Let $p$ be a complex number not in the range of $\phi$. Let $u$ be a complex-valued function on $[a, b]$ such that $(1.1)$ holds. Suppose there is a positive real number $M$ such that $|\phi(t)-p| \geqq M$ for all $t$ in $[a, b]$. Suppose we have a positive real number $\varepsilon$ less than $\pi / 2$ and $a$ partition $\Delta$ of $[a, b]$ such that $\left|\operatorname{im} u\left(t^{\prime \prime}\right)-\operatorname{im} u\left(t^{\prime}\right)\right| \leqq \varepsilon$ whenever $t^{\prime}, t^{\prime \prime}$ are on a closed subinterval of $[a, b]$ determined by $\Delta$. Then, $u$ is of bounded variation on $[a, b]$.

Proof. For each $t$ in $[a, b]$, for simplicity of notation let $\omega(t)=\phi(t)-p$, let $\tilde{\omega}(t)=\operatorname{re} \omega(t)$ and $\hat{\omega}(t)=\operatorname{im} \omega(t)$, and let $\tilde{u}(t)=\operatorname{re} u(t)$ and $\hat{u}(t)=\operatorname{im} u(t)$.

We now show that $\tilde{u}$ is of bounded variation on $[a, b]$. For each $t$ in $[a, b], \tilde{u}(t)$ is the principal logarithm, $\operatorname{Ln}|\omega(t)|$, of the positive real number $|\omega(t)|$. Let $t^{\prime}$ and $t^{\prime \prime}$ be any two real numbers in $[a, b]$. We note that

$$
\left|\tilde{u}\left(t^{\prime \prime}\right)-\tilde{u}\left(t^{\prime}\right)\right|=|\operatorname{Ln}| \omega\left(t^{\prime \prime}\right)|-\operatorname{Ln}| \omega\left(t^{\prime}\right)||=\left|\zeta^{-1}\left\{\left|\omega\left(t^{\prime \prime}\right)\right|-\left|\omega\left(t^{\prime}\right)\right|\right\}\right|
$$

for some $\zeta$ on the segment of the real axis joining $\left|\omega\left(t^{\prime}\right)\right|$ and $\left|\omega\left(t^{\prime \prime}\right)\right|$. Thus,

$$
\left|\tilde{u}\left(t^{\prime \prime}\right)-\tilde{u}\left(t^{\prime}\right)\right| \leqq M^{-1}|| \phi\left(t^{\prime \prime}\right)-p|-| \phi\left(t^{\prime}\right)-p|| \leqq M^{-1}\left|\phi\left(t^{\prime \prime}\right)-\phi\left(t^{\prime}\right)\right| .
$$

From this it follows that $\tilde{u}$ is of bounded variation on $[a, b]$.

Next we consider the more tricky task of showing that the function $\hat{u}$ is of bounded variation on $[a, b]$. Let $\rho$ be a real number satisfying $\varepsilon<\rho<$ $\frac{1}{2}(\varepsilon+\pi / 2)$. For each $t$ in $[a, b], \hat{u}(t)$ is an argument of the nonzero complex number $\omega(t)$. For each $t$ in $[a, b]$ such that $-\pi / 2<\hat{u}(t)<\pi / 2$, we have that

$$
\hat{u}(t)=\int_{0}^{\hat{\omega}(t) /|\omega(t)|}\left(1-s^{2}\right)^{-1 / 2} d s .
$$

For each $t$ in $[a, b]$ such that $0<\hat{u}(t)<\pi$,

$$
\hat{u}(t)=\frac{\pi}{2}-\int_{0}^{\tilde{\omega}(t) /|\omega(t)|}\left(1-s^{2}\right)^{-1 / 2} d s .
$$

For each $t$ in $[a, b]$ such that $\pi / 2<\hat{u}(t)<3 \pi / 2$,

$$
\hat{u}(t)=\pi-\int_{0}^{\hat{\omega}(t) /|\omega(t)|}\left(1-s^{2}\right)^{-1 / 2} d s .
$$

We may obtain analogous expressions for $\hat{u}(t)$ for all $t$ in $[a, b]$. 
Let $t^{\prime}, t^{\prime \prime}$ be real numbers on one of the closed subintervals of $[a, b]$ determined by the partition $\Delta$. Let $z^{\prime}=x^{\prime}+i y^{\prime}$ and $z^{\prime \prime}=x^{\prime \prime}+i y^{\prime \prime}$ denote $\omega\left(t^{\prime}\right)$ and $\omega\left(t^{\prime \prime}\right)$, respectively, in rectangular form. In case $[-\pi / 2+\rho] \leqq$ $\hat{u}\left(t^{\prime}\right) \leqq[\pi / 2-\rho]$, then $[-\pi / 2+(\rho-\varepsilon)] \leqq \hat{u}\left(t^{\prime \prime}\right) \leqq[\pi / 2-(\rho-\varepsilon)]$, so it follows that

$$
\begin{aligned}
\left|\hat{u}\left(t^{\prime \prime}\right)-\hat{u}\left(t^{\prime}\right)\right| & \\
& =\left|\int_{y^{\prime} /\left|z^{\prime}\right|}^{y^{\prime \prime}\left|z^{\prime \prime}\right|}\left(1-s^{2}\right)^{-1 / 2} d s\right| \\
& \leqq \csc (\rho-\varepsilon)\left|\frac{y^{\prime \prime}}{\left|z^{\prime \prime}\right|}-\frac{y^{\prime}}{\left|z^{\prime}\right|}\right|=\csc (\rho-\varepsilon) \frac{\left|y^{\prime \prime}\right| z^{\prime}\left|-y^{\prime}\right| z^{\prime \prime}||}{\left[\left|z^{\prime}\right|\left|z^{\prime \prime}\right|\right]} \\
& =\csc (\rho-\varepsilon) \frac{\left|\left(y^{\prime \prime}-y^{\prime}\right)\right| z^{\prime}\left|+y^{\prime}\left[\left|z^{\prime}\right|-\left|z^{\prime \prime}\right|\right]\right|}{\left[\left|z^{\prime}\right|\left|z^{\prime \prime}\right|\right]} \\
& \leqq \csc (\rho-\varepsilon) \frac{\left[\left|y^{\prime \prime}-y^{\prime}\right|+\left|z^{\prime \prime}-z^{\prime}\right|\right]}{\left|z^{\prime \prime}\right|} \leqq \csc (\rho-\varepsilon) \frac{2\left|z^{\prime \prime}-z^{\prime}\right|}{M} .
\end{aligned}
$$

In case $\hat{u}\left(t^{\prime}\right) \geqq[\pi / 2-\rho]$ and $\hat{u}\left(t^{\prime \prime}\right) \leqq[\pi / 2-\rho]$, then $\hat{u}\left(t^{\prime}\right) \leqq[\pi / 2-(\rho-\varepsilon)]$, and $\hat{u}\left(t^{\prime \prime}\right) \geqq[\pi / 2-\rho]-\varepsilon \geqq[-\pi / 2+(\rho-\varepsilon)]$ since $\rho<\pi / 2$, and so we have as in the preceding case that $\left|\hat{u}\left(t^{\prime \prime}\right)-\hat{u}\left(t^{\prime}\right)\right| \leqq \csc (\rho-\varepsilon) 2\left|z^{\prime \prime}-z^{\prime}\right| \mid M$. In case $[\pi / 2-\rho] \leqq \hat{u}\left(t^{\prime}\right) \leqq[\pi-\rho]$ and $\hat{u}\left(t^{\prime \prime}\right)>[\pi / 2-\rho]$, then $\hat{u}\left(t^{\prime \prime}\right) \leqq[\pi-(\rho-\varepsilon)]$, $\hat{u}\left(t^{\prime}\right) \geqq(\rho-\varepsilon)$ and $\hat{u}\left(t^{\prime \prime}\right) \geqq(\rho-\varepsilon)$ since $[\pi / 2-\rho] \geqq(\rho-\varepsilon)$ in view of the restriction that $\rho \leqq \frac{1}{2}(\varepsilon+\pi / 2)$, and

$$
\begin{aligned}
\left|\hat{u}\left(t^{\prime \prime}\right)-\hat{u}\left(t^{\prime}\right)\right| & =\left|\int_{x^{\prime} /\left|z^{\prime}\right|}^{x^{\prime \prime}\left|z^{\prime \prime}\right|}\left(1-s^{2}\right)^{-1 / 2} d s\right| \\
& \leqq \csc (\rho-\varepsilon)\left|\frac{x^{\prime \prime}}{\left|z^{\prime \prime}\right|}-\frac{x^{\prime}}{\left|z^{\prime}\right|}\right| \leqq \csc (\rho-\varepsilon) \frac{2\left|z^{\prime \prime}-z^{\prime}\right|}{M}
\end{aligned}
$$

as in the first case considered. We may continue thus to show that

$$
\left|\hat{u}\left(t^{\prime \prime}\right)-\hat{u}\left(t^{\prime}\right)\right| \leqq \csc (\rho-\varepsilon) 2 M^{-1}\left|\phi\left(t^{\prime \prime}\right)-\phi\left(t^{\prime}\right)\right| .
$$

Thus, $\hat{u}$ is of bounded variation on $[a, b]$. Hence, $u$ is of bounded variation on $[a, b]$.

This completes the proof of Theorem 3.3.

4. A result relative to the argument principle. Here we show how Theorem 2.1, together with Theorem 3.2, can be used to prove a result of interest in the study of the argument principle. Let $g$ be an analytic complex-valued function in a region $S$ of the complex plane. Let $\phi$ be a continuous complex-valued function of bounded variation on a closed interval $[a, b]$ of the real axis such that the range of $\phi$ is in $S$. Let $p$ be a complex 
number not in the range of $\phi$. Let $\boldsymbol{C}$ be the oriented Fréchet curve for the complex plane containing $(\phi,[a, b])$. Let $\eta(t)=g(\phi(t))$ for all $t$ in $[a, b]$. Clearly $\eta$ is continuous on $[a, b]$, and $\eta$ is of bounded variation on $[a, b]$ in view of Theorem 2.1. Clearly $p$ is not in the range of $\eta$. Let $z^{\prime}=\phi(a)$ and $z^{\prime \prime}=\phi(b)$. Making use of a result in [2] or [3], we let

$$
\begin{aligned}
\int_{C} g^{\prime}(z)[g(z)-p]^{-1} d z & =\int_{a}^{b} g^{\prime}(\phi(t))[g(\phi(t))-p]^{-1} d \phi(t) \\
& =\int_{a}^{b}[\eta(t)-p]^{-1} d \eta(t)
\end{aligned}
$$

by Theorem 2.1. We have from Theorem 3.2 that

$$
\int_{a}^{b}[\eta(t)-p]^{-1} d \eta(t)=\mu(\eta, a, b, p) .
$$

Then, it follows that

$$
\begin{gathered}
\int_{C} g^{\prime}(z)[g(z)-p]^{-1} d z=\left\{\operatorname{Ln}\left|g\left(z^{\prime \prime}\right)-p\right|-\operatorname{Ln}\left|g\left(z^{\prime}\right)-p\right|\right\} \\
+i\{\text { change in a continuous argument of }[g(z)-p] \\
\text { as } z \text { traverses the path determined by } C\} . \\
\text { REFERENCES }
\end{gathered}
$$

\section{REFERENCES}

1. G. T. Whyburn, Topological analysis, Princeton Math. Series, no. 23, Princeton Univ. Press, Princeton, N.J., 1958. MR 20 \#6081.

2. F. M. Wright and A. M. Andronikou, The Weierstrass integral in the complex plane, Bull. Soc. Math. Grèce (1971), 170-190.

3. G. M. Ewing, Calculus of variations with applications, Norton, New York, 1969. MR 39 \#3367.

Department of Mathematics, Iowa State University, Ames, Iowa 50010 